\title{
Fault-Free Hamiltonian Cycles in Faulty Butterfly Graphs
}

\author{
Shien-Ching Hwang and Gen-Huey Chen \\ Department of Computer Science and Information Engineering, \\ National Taiwan University, Taipei, TAIWAN \\ Email: ghchen@csie.ntu.edu.tw
}

\begin{abstract}
The butterfly graphs were originally defined as the underlying graphs of FFT networks which can perform the fast Fourier transform (FFT) very efficiently. Since butterfly graphs are regular of degree four, it can tolerate at most two edge faults in the worst case in order to establish a Hamiltonian cycle. In this paper, we show that butterfly graphs contain a fault-free Hamiltonian cycle even if it has two random edge faults.
\end{abstract}

\section{Introduction}

The butterfly graphs were originally defined as the underlying graphs of FFT networks [7] which can perform the fast Fourier transform (FFT) very efficiently. An excellent description of the butterfly graphs can be found in [10]. The butterfly graphs were known to be an instance of the Cayley graphs (see [2]). The Cayley graphs [1], which represent a category of symmetric and regular graphs derivable from permutation groups, have been shown very suitable to serve as network topologies.

Cycles in networks are useful to many applications such as indexing, embedding linear arrays and rings [9], computing fast Fourier transforms [6], etc. Wong [12] showed that $r$-dimensional $k$-ary butterfly graphs is Hamiltonian-connected if $r$ is odd, and Hamiltonianlaceable if $r$ is even. Barth and Raspaud [3] constructed two edge-disjoint Hamiltonian cycles in $r$-dimensional binary butterfly graphs. Then, Bermond et al. [5] generalized their work by constructing $k$ edge-disjoint Hamiltonian cycles in $r$-dimensional $k$-ary butterfly graphs, where $k \geq 2$. Bermond et al. [4] also constructed edgedisjoint Hamltonian cycles in directed $r$-dimensional $k$-ary butterfly graphs.

Since faults may happen when a network is put in use, it is practically important to consider faulty networks. In this paper, we construct a Hamiltonian cycle in a faulty $r$-dimensional binary butterfly graphs.

The rest of this paper is organized as follows. In the next section, some necessary definitions and notations are introduced and some fundamental properties of butterfly graphs are shown. A Hamiltonian cycle in a faulty butterfly graph is established in Section 3. Finally, this paper concludes with some remarks in Section 4.

\section{Preliminaries}

For convenience we use $B F(r)$ to denote an $r$-dimensional binary butterfly graph. There are $r$ levels, numbered $0,1, \ldots, r-1$, associated with $B F(r)$. Each level contains $2^{r}$ vertices that are labeled with $2^{r}$ binary sequences of $r$ symbols. Hence there are $r 2^{r}$ vertices in $B F(r)$. A vertex of level $l$ labeled with $\beta_{0} \beta_{1} \cdots \beta_{r-1}$ is denoted by $<l, \beta_{0} \beta_{1} \cdots \beta_{r-1}>$, where $0 \leq l \leq r-1$ and $0 \leq \beta_{j} \leq 1$ for all $0 \leq j \leq r-1$. Each vertex $<l, \beta_{0} \beta_{1} \cdots \beta_{r-1}>$ is connected to four vertices: $<l-1 \bmod r, \beta_{0} \beta_{1} \cdots \beta_{l-2} 0 \beta_{l} \cdots \beta_{r-1}>,<l-1$ $\bmod r, \beta_{0} \beta_{1} \cdots \beta_{l-2} 1 \beta_{l} \cdots \beta_{r-1}>,<l+1 \bmod r, \beta_{0} \beta_{1} \cdots \beta_{l-1} 0 \beta_{l+1}$ $\cdots \beta_{r-1}>$, and $<l+1 \bmod r, \beta_{0} \beta_{1} \cdots \beta_{l-1} 0 \beta_{l+1} \cdots \beta_{r-1}>$. In the rest of this paper, we assume that all arithmetic computations with respect to the symbols and the levels are performed modulo 2 and modulo $r$, respectively. A formal definition of $B F(r)$ is as follows.

Definition 1. The vertex set of $B F(r)$ is $\left\{<l, \beta_{0} \beta_{1} \cdots \beta_{r-1}>\mid 0\right.$ $\leq l \leq r-1$ and $0 \leq \beta_{j} \leq 1$ for all $\left.0 \leq j \leq r-1\right\}$. The edge set of $B F(r)$ is $\left\{\left(<l, \beta_{0} \beta_{1} \cdots \beta_{r-1}>,<l+1, \beta_{0}^{\prime} \beta_{1}^{\prime} \cdots \beta_{r-1}>\right) \mid 0 \leq\right.$ $l \leq r-1$ and $\beta_{j}=\beta_{j}^{\prime}$ for all $0 \leq j \leq r-1$ and $\left.j \neq l\right\}$. Moreover, $\left(<l, \beta_{0} \beta_{1} \cdots \beta_{r-1}>,<l+1, \beta_{0}^{\prime} \beta_{1}^{\prime} \cdots \beta_{r-1}>\right)$ is said to be an $i$-edge if $\beta_{l}^{\prime}=\beta_{l}+i$, where $0 \leq i \leq 1$.

Clearly, $B F(r)$ is regular of degree four and its edges exist between adjacent levels. Level 0 and level $r-1$ are considered adjacent. Since $B F(1)$ has trivial structure and $B F(2)$ is a multigraph, we assume $r \geq 3$ throughout this paper. The structure of $B F(3)$ is illustrated in Figure 1, where level 0 is replicated. $B F(r)$ thus defined was named wrapped butterfly graphs in [10].

A cycle $C$ in $B F(r)$ is said to be an $i$-cycle if its edges are all $i$-edges. Suppose $(u, v)$ is an $i$-edge of $B F(r)$, where $u$ is assumed of level $l$ and $v$ is assumed of level $l+1$. Let $f_{i}^{0}(u)=u, f_{i}(u)=v\left(\right.$ or $\left.u=f_{i}^{-1}(v)\right), f_{i}^{j}(u)=f_{i}\left(f_{i}^{j-1}(u)\right)$, and $f_{i}^{-j}(u)=f_{i}^{-1}\left(f_{i}^{-j+1}(u)\right)$ for $j \geq 1$. Then, $\left(u, f_{0}(u)\right.$, $\left.f_{0}^{2}(u), \ldots, f_{0}^{r-1}(u), f_{0}^{r}(u)=u\right)$ is a 0 -cycle, denoted by 
$C_{0}(u)$. We say that $C_{0}(u)$ is the 0 -cycle induced by $u$. Each 0 -cycle in $B F(r)$ has length $r$.

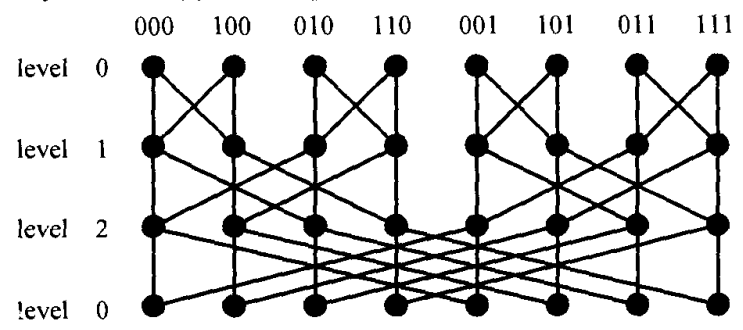

Figure 1. The structure of $B F(3)$ with level 0 replicated.

Suppose $u=\left\langle l, \beta_{0} \beta_{1} \cdots \beta_{r-1}\right\rangle$ and $u^{\prime}=\left\langle l^{\prime}, \beta_{0}^{\prime} \beta_{1}^{\prime} \cdots \beta_{r-1}\right\rangle$ are two distinct vertices of $B F(r)$. It is not difficult to see that $C_{0}(u)=C_{0}\left(u^{\prime}\right)$ if $\beta_{0} \beta_{1} \cdots \beta_{r-1}=\beta_{0}^{\prime} \beta_{1}^{\prime} \cdots \beta_{r-1}{ }^{\prime}$, and $C_{0}(u) \cap$ $C_{0}\left(u^{\prime}\right)$ is empty else. Hence, there are $2^{r}$ vertex-disjoint (disjoint for short) 0 -cycles in $B F(r)$. For each 0 -cycle we assign the vertex of level 0 to be the cycle leader. In subsequent discussion, we use $C_{0}\left(\beta_{0} \beta_{1} \cdots \beta_{r-1}\right)$ to denote the 0 -cycle induced by $<0, \beta_{0} \beta_{1} \cdots \beta_{r-1}>$. We say that two disjoint cycles in $B F(r)$ are adjacent if there exists an edge between them.

Lemma 1. In $B F(r), C_{0}\left(\beta_{0} \beta_{1} \cdots \beta_{r-1}\right)$ is adjacent to $r$ disjoint 0 -cycles, i.e., $C_{0}\left(\beta_{0} \beta_{1} \cdots \beta_{j-1}\left(\beta_{j}+i\right) \beta_{j+1} \cdots \beta_{r-1}\right)$ for all $1 \leq i \leq$ 1 and $0 \leq j \leq r-1$.

Proof. Suppose $C_{0}\left(\beta_{0} \beta_{1} \cdots \beta_{r-1}\right)=\left(\varepsilon, \quad f_{0}(\varepsilon), \quad f_{0}^{2}(\varepsilon), \ldots\right.$, $\left.f_{0}^{r-1}(\varepsilon), f_{0}^{r}(\varepsilon)=\varepsilon\right)$, where $\varepsilon=<0, \beta_{0} \beta_{1} \cdots \beta_{r-1}>$. There are $2 r$ vertices of $B F(r)$ that are adjacent to one vertex of $C_{0}\left(\beta_{0} \beta_{1} \cdots \beta_{r-1}\right)$ but not contained in $C_{0}\left(\beta_{0} \beta_{1} \cdots \beta_{r-1}\right)$. These $2 r$ vertices can induce $2 r$-cycles that are adjacent to $C_{0}\left(\beta_{0} \beta_{1} \cdots \beta_{r-1}\right)$. In the following, we show that $r$ of them are disjoint.

Since $f_{1}(\varepsilon)=<1,\left(\beta_{0}+1\right) \beta_{1} \cdots \beta_{r-1}>$ and $f_{1}\left(f_{0}^{j}(\varepsilon)\right)=<j+$ 1, $\beta_{0} \beta_{1} \cdots \beta_{j-1}\left(\beta_{j}+1\right) \beta_{j+1} \cdots \beta_{r-1}>, C_{0}\left(f_{1}(\varepsilon)\right)$ has cycle leader $<0,\left(\beta_{0}+1\right) \beta_{1} \cdots \beta_{r-1}>$ and $C_{0}\left(f_{1}\left(f_{0}^{j}(\varepsilon)\right)\right)$ has cycle leader $<0, \beta_{0} \beta_{1} \cdots \beta_{j-1}\left(\beta_{j}+1\right) \beta_{j+1} \cdots \beta_{r-1}>$, where $1 \leq j \leq r-1$. Similarly, $C_{0}\left(f_{1}^{-1}(\varepsilon)\right)$ and $C_{0}\left(f_{1}^{-1}\left(f_{0}^{j}(\varepsilon)\right)\right)$ have cycle leaders $<0, \beta_{0} \beta_{1} \cdots\left(\beta_{r-1}-1\right)>$ and $<0, \beta_{0} \beta_{1} \cdots \beta_{j-2}\left(\beta_{j-1}-\right.$ 1) $\beta_{j} \cdots \beta_{r-1}>$, respectively. It is not difficult to see that all different cycle leaders form the set $\left\{<0, \beta_{0} \beta_{1} \cdots \beta_{j-1}\left(\beta_{j}+\right.\right.$ 1) $\left.\beta_{j+1} \cdots \beta_{r-1}>\mid 0 \leq j \leq r-1\right\}$ whose size is $r$. These different cycle leaders can induce $r$ disjoint 0 -cycles.

Suppose $C$ and $C^{\prime}$ are two adjacent cycles in $B F(r)$, and $\left(u, v^{\prime}\right)$ and $\left(v, u^{\prime}\right)$ are two edges between $C$ and $C^{\prime}$. Then $(u$, $\left.v^{\prime}\right)$ and $\left(v, u^{\prime}\right)$ are said to be a crossing pair of level $l$ between $C$ and $C^{\prime}$ if $(u, v)$ and $\left(u^{\prime}, v^{\prime}\right)$ are two edges of $C$ and $C^{\prime}$, respectively, between levels $l$ and $l+1$. In subsequent discussion, we define $\operatorname{dif}\left(\beta_{0} \beta_{1} \cdots \beta_{r-1}, \beta_{0}{ }^{\prime} \beta_{1}{ }^{\prime} \cdots\right.$ $\left.\beta_{r-1}\right)=j$ if $\beta_{j} \neq \beta_{j}{ }^{\prime}$ and $\beta_{i}=\beta_{i}{ }^{\prime}$ for all $0 \leq i \leq r-1$ and $i \neq j$.
A condensation of $B F(r)$, denoted by $C B F_{0}(r)$, is defined as follows. Each 0 -cycle $C_{0}\left(\beta_{0} \beta_{1} \cdots \beta_{r-1}\right)$ in $B F(r)$ corresponds to a vertex $\beta_{0} \beta_{1} \cdots \beta_{r-1}$ of $C B F_{0}(r)$, and every pair of adjacent 0 -cycles in $B F(r)$ correspond to an edge of $C B F_{0}(r)$. That is, $C B F_{0}(r)$ has vertex set $\left\{\beta_{0} \beta_{1} \cdots \beta_{r-1} \mid \beta_{j}\right.$ $\in\{0,1\}$ for all $0 \leq j \leq r-1\}$ and edge set $\left\{\left(\beta_{0} \beta_{1} \cdots \beta_{r-1}\right.\right.$, $\left.\left.\beta_{0} \beta_{1} \cdots \beta_{j-1}\left(\beta_{j}+1\right) \beta_{j+1} \cdots \beta_{r-1}\right) \mid 0 \leq j \leq r-1\right\}$ according to Lemma 1. We note that $C B F_{0}(r)$ is isomorphic to the $r$-dimensional hypercube [11]. Each path $\left(x_{1}, x_{2}, \ldots, x_{m}\right)$ in $C B F_{0}(r)$ has $\operatorname{dif}\left(x_{i-1}, x_{i}\right) \neq \operatorname{dif}\left(x_{i}, x_{i+1}\right)$ for all $2 \leq i \leq m-1$.

Lemma 2. Suppose $C_{0}(x)$ and $C_{0}(y)$ are two adjacent 0 -cycles in $B F(r)$. There are exactly two edges between them which form a crossing pair of level $\operatorname{dif}(x, y)$.

Proof. Suppose diff $x, y)=l$, and assume $x=\beta_{0} \beta_{1} \cdots \beta_{r-1}$ and $y=\beta_{0} \beta_{1} \cdots \beta_{l-1} \beta_{l}^{\prime} \beta_{l+1} \cdots \beta_{r-1}$, where $\beta_{l} \neq \beta_{l}^{\prime}$. There are two edges $\left(<l, \beta_{0} \beta_{1} \cdots \beta_{r-1}>,<l+1, \beta_{0} \beta_{1} \cdots \beta_{l-1} \beta_{l}^{\prime} \beta_{l+1} \cdots \beta_{r-1}>\right)$ and $\left(<l+1, \beta_{0} \beta_{1} \cdots \beta_{r-1}>,<l, \beta_{0} \beta_{1} \cdots \beta_{i-1} \beta_{l}^{\prime} \beta_{l+1} \cdots \beta_{r-1}>\right)$ between $C_{0}(x)$ and $C_{0}(y)$, which form a crossing pair of level $l$. There is no other edge between $C_{0}(x)$ and $C_{0}(y)$.

Lemma 3. A path $\left(x_{1}, x_{2}, \ldots, x_{m}\right)$ of length $m-1$ in $C B F_{0}(r)$ corresponds to a cycle of length $m r$ in $B F(r)$ which contains the vertices of $C_{0}\left(x_{1}\right), C_{0}\left(x_{2}\right), \ldots, C_{0}\left(x_{m}\right)$, where 2 $\leq m \leq 2^{r}$.

Proof. According to Lemma 2, there is a unique crossing pair of level $\operatorname{dif}\left(x_{1}, x_{2}\right)$ between $C_{0}\left(x_{1}\right)$ and $C_{0}\left(x_{2}\right)$. We assume that $\left(u_{1}, v_{2}\right)$ and $\left(v_{1}, u_{2}\right)$ are the crossing pair, where $u_{1}$ and $u_{2}$ are of level $\operatorname{dif}\left(x_{1}, x_{2}\right)$ and $v_{1}$ and $v_{2}$ are of level $\operatorname{dif}\left(x_{1}, x_{2}\right)+1$. There is a cycle of length $2 r$ in $B F(r)$ which contains the crossing pair and all the edges of $C_{0}\left(x_{1}\right)$ and $C_{0}\left(x_{2}\right)$ but $\left(u_{1}, v_{1}\right)$ and $\left(u_{2}, v_{2}\right)$.

Similarly, there is a unique crossing pair, say $\left(w_{2}, t_{3}\right)$ and $\left(t_{2}, w_{3}\right)$, of level $\operatorname{dif}\left(x_{2}, x_{3}\right)$ between $C_{0}\left(x_{2}\right)$ and $C_{0}\left(x_{3}\right)$, where $w_{2}$ and $w_{3}$ are assumed of level dif $\left(x_{2}, x_{3}\right)$ and $t_{2}$ and $t_{3}$ are assumed of level $\operatorname{dif}\left(x_{2}, x_{3}\right)+1$. Refer to Figure 2 . Since $\operatorname{dif}\left(x_{1}, x_{2}\right) \neq \operatorname{dif}\left(x_{2}, x_{3}\right)$, there is a cycle of length $3 r$ in $B F(r)$ which contains the crossing pair $\left(w_{2}, t_{3}\right)$ and $\left(t_{2}, w_{3}\right)$, the crossing pair $\left(u_{1}, v_{2}\right)$ and $\left(v_{1}, u_{2}\right)$, and all the edges of $C_{0}\left(x_{1}\right), C_{0}\left(x_{2}\right)$, and $C_{0}\left(x_{3}\right)$ but $\left(u_{1}, v_{1}\right),\left(u_{2}, v_{2}\right),\left(w_{2}, t_{2}\right)$, and $\left(w_{3}, t_{3}\right)$. The merging can proceed with $C_{0}\left(x_{4}\right), C_{0}\left(x_{5}\right), \ldots$, $C_{0}\left(x_{m}\right)$, sequentially, and finally, a cycle of length $m r$ in $B F(r)$ can result.

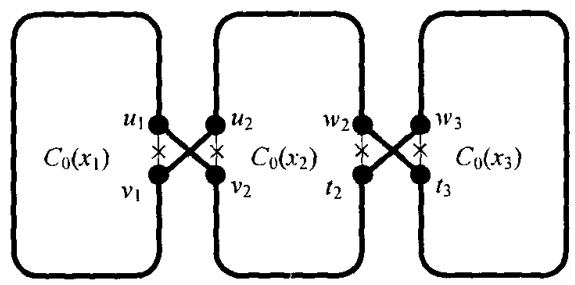

Figure 2. Constructing a cycle of length $3 r$ by merging $C_{0}\left(x_{1}\right), C_{0}\left(x_{2}\right)$, and $C_{0}\left(x_{3}\right)$. 
Suppose $u=<l, \beta_{0} \beta_{1} \cdots \beta_{r-1}>$ is a vertex of $B F(r)$. Then $\left(u, f_{1}(u), f_{1}^{2}(u), \ldots, f_{1}^{2 r}(u)\right)$ is a 1 -cycle, denoted by $C_{1}(u)$. We say that $C_{1}(u)$ is the 1 -cycle induced by $u$. Each 1 -cycle in $B F(r)$ has length $2 r$.

Suppose $u=\left\langle l, \beta_{0} \beta_{1} \cdots \beta_{r-1}>\right.$ and $u^{\prime}=\left\langle l, \beta_{0}{ }^{\prime} \beta_{1}^{\prime} \cdots \beta_{r-1}\right\rangle$ are two distinct vertices of $B F(r)$. It is not difficulty to see that $C_{1}(u)=C_{1}\left(u^{\prime}\right)$ if they have vertices in common. Hence, there are $2^{r-1}$ disjoint 1 -cycles in $B F(r)$. Since $f_{1}^{\prime r}(u)=<l$, $\left(\beta_{0}+t\right)\left(\beta_{1}+t\right) \cdots\left(\beta_{r-1}+t\right)>$ for integer $t \geq 1, C_{1}(u)=C_{1}\left(u^{\prime}\right)$ if and only if $\beta_{j}^{\prime}=\beta_{j}+1$ for all $0 \leq j \leq r-1$. The following lemma is clear, and so the proof is omitted.

Lemma 4. Suppose $C_{1}(u)$ and $C_{1}(v)$ are two adjacent 1-cycles in $B F(r)$, and $u_{0} \in C_{1}(u)$ is adjacent to $v_{0} \in C_{1}(v)$. Then, $f_{1}^{r}\left(u_{0}\right) \in C_{1}(u)$ is adjacent to $f_{1}^{r}\left(v_{0}\right) \in C_{1}(v)$.

Lemma 5. Suppose $C_{1}(u)$ and $C_{1}(v)$ are two adjacent 1 -cycles in $B F(r)$, and $u_{0} \in C_{1}(u)$ is adjacent to $v_{0} \in C_{1}(v)$. There are two adjacent vertices $u_{0}{ }^{\prime} \in C_{1}(u)$ and $v_{0}{ }^{\prime} \in C_{1}(v)$ so that $u_{0}{ }^{\prime}$ is adjacent to $u_{0}$ and $v_{0}{ }^{\prime}$ is adjacent to $v_{0}$.

Proof. Suppose $u_{0}=<l, \beta_{0} \beta_{1} \cdots \beta_{r-1}>$ and $v_{0}=f_{0}\left(u_{0}\right)=<l+1$, $\beta_{0} \beta_{1} \cdots \beta_{r-1}>$. We let $u_{0}{ }^{\prime}=f_{1}\left(u_{0}\right)=<l+1, \beta_{0} \beta_{1} \cdots \beta_{l-1}\left(\beta_{l}+\right.$ 1) $\beta_{l+1} \cdots \beta_{r-1}>\in C_{1}(u)$ and $v_{0}{ }^{\prime}=f_{1}^{-1}\left(v_{0}\right)=<l, \beta_{0} \beta_{1} \cdots \beta_{l-1}\left(\beta_{l}\right.$ $-1) \beta_{l+1} \cdots \beta_{r-1}>\in C_{1}(v)$. Since $u_{0}{ }^{\prime}=f_{0}\left(v_{0}\right), u_{0}{ }^{\prime}$ is adjacent to $v_{0}^{\prime}$.

Lemma 6. There are 4 edges between every two adjacent 1-cycles in $B F(r)$.

Proof. Suppose $C_{1}(u)$ and $C_{1}(v)$ are two adjacent 1-cycles in $B F(r)$, and $u_{0} \in C_{1}(u)$ is adjacent to $v_{0} \in C_{1}(v)$. According to Lemma 5, there are two adjacent vertices $u_{0}{ }^{\prime}$ $\in C_{1}(u)$ and $v_{0}{ }^{\prime} \in C_{1}(v)$ so that $u_{0}{ }^{\prime}$ is adjacent to $u_{0}$ and $v_{0}{ }^{\prime}$ is adjacent to $v_{0}$. According to Lemma $4, f_{1}^{r}\left(u_{0}\right) \in C_{1}(u)$ is adjacent to $f_{1}^{r}\left(v_{0}\right) \in C_{1}(v)$ and $f_{1}^{r}\left(u_{0}\right) \in C_{1}(u)$ is adjacent to $f_{1}^{r}\left(v_{0}\right) \in C_{1}(v)$. Hence, there are 4 edges between $C_{1}(u)$ and $C_{1}(v)$.

For each 1-cycle we assign the vertex of level 0 whose leftmost symbol is 0 to be the cycle leader. For example, the 1 -cycle $(<0,001\rangle,<1,101\rangle,<2,111>,<0,110\rangle,<1$, $010>,<2,000>,<0,001>)$ in $B F(3)$ has cycle leader $<0$, $001>$. In subsequent discussion, we use $C_{1}\left(0 \beta_{1} \beta_{2} \cdots \beta_{r-1}\right)$ to denote the 1-cycle induced by $<0,0 \beta_{1} \beta_{2} \cdots \beta_{r-1}>$.

Lemma 7. In $B F(r), C_{1}\left(0 \beta_{1} \beta_{2} \cdots \beta_{r-1}\right)$ is adjacent to $r$ disjoint 1-cycles, i.e., $C_{1}\left(0\left(\beta_{1}+1\right)\left(\beta_{2}+1\right) \cdots\left(\beta_{r-1}+1\right)\right)$ and $C_{1}\left(0 \beta_{1} \beta_{2} \cdots \beta_{j-1}\left(\beta_{j}+1\right) \beta_{j+1} \cdots \beta_{r-1}\right)$ for all $1 \leq j \leq r-1$.

Proof. Suppose $u=<l, \beta_{0} \beta_{1} \cdots \beta_{r-1}>\in C_{1}\left(0 \beta_{1} \beta_{2} \cdots \beta_{r-1}\right)$. There are two vertices of $B F(r)$ that are adjacent to $u$ but not contained in $C_{1}\left(0 \beta_{1} \beta_{2} \cdots \beta_{r-1}\right)$. These two vertices can induce two 1-cycles which are all adjacent to
$C_{1}\left(0 \beta_{1} \beta_{2} \cdots \beta_{r-1}\right)$. We use $S$ to denote the set of these two 1-cycles. Similarly, there are two vertices of $B F(r)$ that are adjacent to $f_{1}^{r}(u)=<l,\left(\beta_{0}+1\right)\left(\beta_{1}+1\right) \cdots\left(\beta_{r-1}+1\right)>$ but not contained in $C_{1}\left(0 \beta_{1} \beta_{2} \cdots \beta_{r-1}\right)$. We use $T$ to denote the set of the two 1 -cycles that are induced by the two vertices. Recall that $C_{1}(u)=C_{1}\left(u^{\prime}\right)$ if and only if $\beta_{j}{ }^{\prime}=\beta_{j}+1$ for all 0 $\leq j \leq r-1$, where $u^{\prime}=<l, \beta_{0}{ }^{\prime} \beta_{1}{ }^{\prime} \cdots \beta_{r-1}>$ is a vertex of $B F(r)$ and $u^{\prime} \neq u$. We have $S=T$. Hence, it suffices to consider only $r$ consecutive vertices of $C_{1}\left(0 \beta_{1} \beta_{2} \cdots \beta_{r-1}\right)$, say $\varepsilon, f_{1}(\varepsilon)$, $f_{1}^{2}(\varepsilon), \ldots, f_{\mathrm{l}}^{r-1}(\varepsilon)$, where $\varepsilon=<0,0 \beta_{1} \beta_{2} \cdots \beta_{r-1}>$.

There are $2 r$ vertices of $B F(r)$ that are adjacent to one of $\varepsilon, f_{1}(\varepsilon), f_{1}^{2}(\varepsilon), \ldots, f_{1}^{r-1}(\varepsilon)$ but not contained in $C_{1}\left(0 \beta_{1} \beta_{2} \cdots \beta_{r-1}\right)$. They are $f_{0}(\varepsilon), f_{0}^{-1}(\varepsilon), f_{0}\left(f_{1}(\varepsilon)\right)$, $f_{0}^{-1}\left(f_{1}(\varepsilon)\right), f_{0}\left(f_{1}^{2}(\varepsilon)\right), f_{0}^{-1}\left(f_{1}^{2}(\varepsilon)\right), \ldots, f_{0}\left(f_{1}^{r-1}(\varepsilon)\right)$, $f_{0}^{-1}\left(f_{1}^{r-1}(\varepsilon)\right)$. Since $f_{1}^{r-1}\left(f_{0}(\varepsilon)\right)=<0,0\left(\beta_{1}+1\right)\left(\beta_{2}+1\right) \cdots$ $\left(\beta_{r-1}+1\right)>$ and $f_{1}^{-(j+1)}\left(f_{0}\left(f_{1}^{j}(\varepsilon)\right)\right)=<0,0 \beta_{1} \beta_{2} \cdots\left(\beta_{j}-1\right)$ $\beta_{j+1} \cdots \beta_{r-1}>, C_{1}\left(f_{0}(\varepsilon)\right)$ has cycle leader $<0,0\left(\beta_{1}+1\right)\left(\beta_{2}+1\right)$ $\cdots\left(\beta_{r-1}+1\right)>$ and $C_{1}\left(f_{0}\left(f_{1}^{j}(\varepsilon)\right)\right)$ has cycle leader $<0,0 \beta_{1} \beta_{2}$ $\cdots\left(\beta_{j}-1\right) \beta_{j+1} \cdots \beta_{r-1}>$, where $1 \leq j \leq r-1$.

Similarly, $C_{1}\left(f_{0}^{-1}(\varepsilon)\right)$ has cycle leader $<0,0 \beta_{1} \beta_{2} \cdots \beta_{r-2}$ $\left(\beta_{r-1}+1\right)>, C_{1}\left(f_{0}^{-1}\left(f_{1}(\varepsilon)\right)\right)$ has cycle leader $<0,0\left(\beta_{1}-1\right)$ $\left(\beta_{2}-1\right) \cdots\left(\beta_{r-1}-1\right)>$, and $C_{1}\left(f_{0}^{-1}\left(f_{1}^{j}(\varepsilon)\right)\right)$ has cycle leader $<0,0 \beta_{1} \beta_{2} \cdots \beta_{j-2}\left(\beta_{j-1}+1\right) \beta_{j} \cdots \beta_{r-1}>$, where $2 \leq j \leq r-1$. All the cycle leaders constitute the following set.

$$
\begin{aligned}
W= & \left\{<0,0\left(\beta_{1}+1\right)\left(\beta_{2}+1\right) \cdots\left(\beta_{r-1}+1\right)>\right\} \cup \\
& \left\{<0,0 \beta_{1} \beta_{2} \cdots \beta_{j-1}\left(\beta_{j}+1\right) \beta_{j+1} \cdots \beta_{r-1}>\mid 1 \leq j \leq r-1\right\} .
\end{aligned}
$$

The lemma follows because $|W|=r$.

A condensation of $B F(r)$, denoted by $C B F_{1}(r)$, is defined as follows. Each 1-cycle $C_{1}\left(0 \beta_{1} \beta_{2} \cdots \beta_{r-1}\right)$ in $B F(r)$ corresponds to a vertex $\beta_{1} \beta_{2} \cdots \beta_{r-1}$ of $C B F_{1}(r)$, and every pair of adjacent 1-cycles in $B F(r)$ correspond to an edge of $C B F_{1}(r)$. That is, $C B F_{1}(k, r)$ has vertex set $\left\{\beta_{1} \beta_{2} \cdots \beta_{r-1} \mid 0 \leq\right.$ $\beta_{i} \leq 1$ for all $\left.1 \leq i \leq r-1\right\}$ and edge set $\left\{\left(\beta_{1} \beta_{2} \cdots \beta_{r-1}, \beta_{1} \beta_{2} \cdots\right.\right.$ $\left.\beta_{i-1}\left(\beta_{i}+1\right) \beta_{i+1} \cdots \beta_{r-1}\right),\left(\beta_{1} \beta_{2} \cdots \beta_{r-1,}\left(\beta_{1}+1\right)\left(\beta_{2}+1\right) \cdots\left(\beta_{r-1}+\right.\right.$ 1)) $\{1 \leq i \leq r-1\}$ according to Lemma 7 . We note that the $(r-1)$-dimensional hypercube is a spanning subgraph of $C B F_{1}(r)$.

Lemma 8. A path of length $n-1$ in $C B F_{1}(r)$ corresponds to a cycle of length $2 n r$ in $B F(r)$.

Proof. Suppose $\left(x_{1}, x_{2}, \ldots, x_{n}\right)$ is a path of length $n-1$ in $C B F_{1}(r)$. According to Lemma 5, there exist two adjacent vertices $u_{i-1}, v_{i-1}$ of $C_{1}\left(x_{i-1}\right)$ and two adjacent vertices $u_{i}, v_{i}$ of $C_{1}\left(x_{i}\right)$ so that $u_{i-1}$ and $v_{i-1}$ are adjacent to $u_{i}$ and $v_{i}$, respectively, where $2 \leq i \leq n-1$. Similarly, there exist two adjacent vertices $u_{i}^{\prime}, v_{i}^{\prime}$ of $C_{1}\left(x_{i}\right)$ and two adjacent vertices 
$u_{i+1}, v_{i+1}$ of $C_{1}\left(x_{i+1}\right)$ so that $u_{i}{ }^{\prime}$ and $v_{i}{ }^{\prime}$ are adjacent to $u_{i+1}$ and $v_{i+1}$, respectively. It is assured by Lemma 6 that $u_{i}^{\prime} \notin\left\{u_{i}, v_{i}\right\}$ and $v_{i}{ }^{\prime} \notin\left\{u_{i}, v_{i}\right\}$ can be determined. A cycle of length $2 n r$ in $B F(r)$ can be constructed as shown in Figure 3.

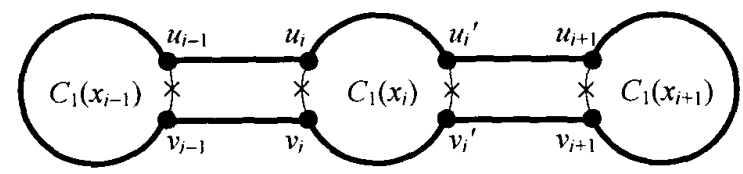

Figure 3. Constructing a cycle of length $n k r$ in $B F(r)$.

A Hamiltonian cycle of $C B F_{1}(r)$ can be obtained using Grad code. a cyclically ordered sequence of $2^{d}$ distinct binary codewords forms a d-dimensional Grad code, denoted by $G(d)$, provided every two adjacent codewords differ in exactly one dimension [8], where $d \geq 1 . G(d)$ can be constructed recursively as follows. Initially, let $G(1)=(0$, 1). For $i \geq 2, G(i)=\left(0 G(i-1), 1 G^{R}(i-1)\right)$, where $G^{R}(i-1)$ denotes the reverse of $G(i-1)$. For example, $G(2)=$ $\left(0 G(1), 1 G^{R}(1)\right)=(00,01,11,10)$ and $G(3)=(0 G(2)$, $\left.1 G^{R}(2)\right)=(000,001,011,010,110,111,101,100)$.

Since $G(r-1)$ contains all the vertices of the $(r-$ 1)-dimensional hypercube and every two adjacent codewords of $G(r-1)$ correspond to an edge of the $(r-$ 1)-dimensional hypercube, $G(r-1)$ corresponds to a Hamiltonian cycle in the $(r-1)$-dimensional hypercube. Thus, $G(r-1)$ corresponds to a Hamiltonian cycle in $C B F_{1}(r)$.

\section{Hamiltonian cycles in faulty $B F(r)$}

Since $B F(r)$ is regular of degree four, it can tolerate at most two edge faults in the worst case in order to establish a Hamiltonian cycle. In this section, we show that $B F(r)$ contains a Hamiltonian cycle even if it has two edge faults.

We observe that $C B F_{0}(r)$, which is isomorphic to the $r$-dimensional hypercube, is recursive. Suppose $e=\left(\beta_{0} \beta_{1} \cdots\right.$ $\left.\beta_{r-1}, \beta_{0}^{\prime} \beta_{1}^{\prime} \cdots \beta_{r-1}\right)$ is an edge of $C B F_{0}(r)$, where $\operatorname{dif}\left(\beta_{0} \beta_{1} \cdots\right.$ $\left.\beta_{r-1}, \beta_{0}^{\prime} \beta_{1}^{\prime} \cdots \beta_{r-1}\right)=q$ is assumed. The two subsets of vertices with $\beta_{q}=0$ and $\beta_{q}=1$, respectively, form a partition of the vertex set of $C B F_{0}(r)$. The two subgraphs of $C B F_{0}(r)$ that are induced by the two subsets are isomorphic to $C B F_{0}(r-1)$. In subsequent discussion, we let dif(e) $=$ $\operatorname{dif}\left(\beta_{0} \beta_{1} \cdots \beta_{r-1}, \quad \beta_{0}^{\prime} \beta_{1}^{\prime} \cdots \beta_{r-1}\right)$ and use $H_{e}^{0}$ and $H_{e}^{1}$ to denote the two subgraphs, respectively. The following three lemmas state fundamental properties of $C B F_{0}(r)$.

Lemma 9. Suppose $e_{1}$ and $e_{2}$ are two distinct edges of $C B F_{0}(r)$. There exists a Hamiltonian cycle in $C B F_{0}(r)$ that contains $e_{1}$ and $e_{2}$.

Proof. We first show by induction on $r$ that given an arbitrary edge $e$ of $C B F_{0}(r)$, there is a Hamiltonian cycle in
$C B F_{0}(r)$ that contains $e$. Since $C B F_{0}(3)$ is isomorphic to the three-dimensional hypercube, our claim is true for $C B F_{0}(3)$. We then assume that our claim is true for $C B F_{0}(r-1)$, where $r-1 \geq 3$.

Suppose $e=\left(v_{1}, v_{2}\right)$ is an edge of $C B F_{0}(r)$, where $v_{1} \in$ $H_{c}^{0}$ and $v_{2} \in H_{e}^{1}$. There exist $v_{1}^{\prime} \in H_{e}^{0}$ and $v_{2}^{\prime} \in H_{e}^{1}$ so that $v_{1}^{\prime}$ is adjacent to $v_{1}, v_{2}{ }^{\prime}$ is adjacent to $v_{2}$, and $v_{1}{ }^{\prime}$ is adjacent to $v_{2}{ }^{\prime}$. By our assumption, there are a Hamiltonian cycle in $H_{e}^{0}$ that contains the edge $\left(v_{1}, v_{1}\right)$ and a Hamiltonian cycle in $H_{e}^{1}$ that contains the edge $\left(v_{2}, v_{2}\right)$. Since $e$ and $\left(v_{1}^{\prime}, v_{2}\right)$ are two edges between the two Hamiltonian cycles, there is a Hamiltonian cycle in $C B F_{0}(r)$ that contains $e,\left(v_{1}{ }^{\prime}, v_{2}\right)$, and all the edges of the two Hamiltonian cycles but $\left(v_{1}, v_{1}\right)$ and $\left(v_{2}, v_{2}\right)$.

Suppose $q=\operatorname{dif}\left(e_{1}\right)$ and $\left(x_{1}, x_{2}, \ldots, x_{2^{r-1}}, x_{1}\right)$ is a Hamiltonian cycle in $H_{e_{1}}^{0}$. Then, $\left(x_{1}^{(q)}, x_{2}^{(q)}, \ldots, x_{2^{-1}}^{(q)}\right.$, $\left.x_{1}^{(q)}\right)$ is a Hamiltonian cycle in $H_{e_{1}}^{1}$. If $\operatorname{dif}\left(e_{2}\right)=\operatorname{dif}\left(e_{1}\right)$, then $\left(x_{1}, x_{1}^{(q)}, x_{2}^{(q)}, x_{2}, x_{3}, x_{3}^{(q)}, x_{4}^{(q)}, \ldots, x_{2^{\prime-1}-1}^{(q)}, x_{2^{\prime-1}}^{(q)}\right.$, $\left.x_{2^{-1}}, x_{1}\right)$ is a Hamiltonian cycle in $C B F_{0}(r)$ that contains $e_{1}$ and $e_{2}$. If dif $\left(e_{2}\right) \neq \operatorname{dif}\left(e_{1}\right)$, we assume $e_{2} \in H_{e_{1}}^{0}$ without loss of generality. There is a Hamiltonian cycle, say $\left(y_{1}, y_{2}, \ldots\right.$, $\left.y_{2^{\prime-1}}, y_{1}\right)$, in $H_{e_{1}}^{0}$ that contains $e_{2}$. Suppose $e_{1}=\left(y_{s}, y_{s}^{(q)}\right)$ for some $1 \leq s \leq 2^{r-1}$ and $e_{2}=\left(y_{i}, y_{i+1}\right)$ for some $1 \leq t \leq 2^{r-1}$. A Hamiltonian cycle in $C B F_{0}(r)$ that contains $e_{1}$ and $e_{2}$ can be established as $\left(y_{s+1}, y_{s+2}, \ldots, y_{2^{r-1}}, y_{1}, y_{2}, \ldots, y_{s}, y_{s}^{(q)}\right.$, $\left.y_{s-1}^{(q)}, \ldots, y_{1}^{(q)}, y_{2^{r-1}}^{(q)}, y_{2^{r-1}-1}^{(q)}, \ldots, y_{s+1}^{(q)}, y_{s+1}\right)$ if $s \neq t$, and $\left(y_{s}^{(q)}, y_{s}, y_{s+1}, \ldots, y_{2^{-4}}, y_{1}, y_{2}, \ldots, y_{s-1}, y_{s-1}^{(q)}, y_{s-2}^{(q)}, \ldots\right.$, $\left.y_{1}^{(q)}, y_{2^{\prime-1}}^{(q)}, y_{2^{2-1}-1}^{(q)}, \ldots, y_{s+1}^{(q)}, y_{s}^{(q)}\right)$ if $s=t$.

Lemma 10. Suppose $e_{1}$ and $e_{2}$ are two distinct edges of $C B F_{0}(r)$. There exists a Hamiltonian path in $C B F_{0}(r)$ that does not contain $e_{1}$ and $e_{2}$.

Proof. Suppose $q=\operatorname{dif}\left(e_{1}\right)$ and $\left(x_{1}, x_{2}, \ldots, x_{2^{r-1}}, x_{1}\right)$ is a Hamiltonian cycle in $H_{e_{1}}^{0}$. If dif $\left(e_{2}\right)=\operatorname{dif}\left(e_{1}\right)$, determine an edge $\left(x_{s}, x_{s}^{(q)}\right) \notin\left\{e_{1}, e_{2}\right\}$ for some $1 \leq s \leq 2^{r-1}$. A Hamiltonian path in $C B F_{0}(r)$ that does not contain $e_{1}$ and $e_{2}$ can be established as $\left(x_{s-1}^{(q)}, x_{s-2}^{(q)}, \ldots, x_{1}^{(q)}, x_{2^{r-1}}^{(q)}\right.$; $\left.x_{2^{r-1}-1}^{(q)}, \ldots, x_{s}^{(q)}, x_{s}, x_{s+1}, \ldots, x_{2^{r-1}}, x_{1}, x_{2}, \ldots, x_{s-1}\right)$. If dif $\left(e_{2}\right)$ $\neq$ dif $\left(e_{1}\right)$, we assume $e_{2} \in H_{e_{1}}^{0}$ without loss of generality. There is a Hamiltonian cycle, say $\left(y_{1}, y_{2}, \ldots, y_{2^{r-1}}, y_{1}\right)$, in $H_{e_{1}}^{0}$ that contains $e_{2}$. Suppose $e_{2}=\left(y_{t}, y_{t+1}\right)$ for some $1 \leq t$ $\leq 2^{r-1}$. A Hamiltonian path in $C B F_{0}(r)$ that does not contain 
$e_{1}$ and $e_{2}$ can be established as $\left(y_{t+1}, y_{1+2}, \ldots, y_{2^{\prime-1}}, y_{1}\right.$, $\left.y_{2}, \ldots, y_{t}, y_{1}^{(q)}, y_{i-1}^{(q)}, \ldots, y_{1}^{(q)}, y_{2^{-1}}^{(q)}, y_{2^{r-1}-1}^{(q)}, \ldots, y_{t+1}^{(q)}\right)$ if $e_{1} \neq\left(y_{t}, y_{t}^{(q)}\right)$, and $\left(y_{t}, y_{t-1}, \ldots, y_{1}, y_{2^{r-1}}, y_{2^{r-1}-1}, \ldots, y_{t+1}\right.$, $\left.y_{t+1}^{(q)}, y_{t+2}^{(q)}, \ldots, y_{2^{r-1}}^{(q)}, y_{1}^{(q)}, y_{2}^{(q)}, \ldots, y_{1}^{(q)}\right)$ if $e_{1}=\left(y_{l}\right.$, $\left.y_{1}^{(i)}\right)$.

Lemma 11. Suppose $e_{1}$ and $e_{2}$ are two distinct edges of $C B F_{0}(r)$. There exists a Hamiltonian path in $C B F_{0}(r)$ that contains $e_{1}$ but does not contain $e_{2}$.

Proof. Suppose $q=\operatorname{diff}\left(e_{1}\right)$ and $\left(x_{1}, x_{2}, \ldots, x_{2^{r-1}}, x_{1}\right)$ is a Hamiltonian cycle in $H_{e_{1}}^{0}$. Then $e_{1}=\left(x_{s}, x_{s}^{(q)}\right)$ for some 1 $\leq s \leq 2^{r-1}$. If diff(e $\left.e_{2}\right)=\operatorname{dif}\left(e_{1}\right)$, a Hamiltonian path in $C B F_{0}(r)$ that contains $e_{1}$ but does not contain $e_{2}$ can be established as $\left(x_{s+1}, x_{s+2}, \ldots, x_{2^{r-1}}, x_{1}, x_{2}, \ldots, x_{s}, x_{s}^{(q)}, x_{s-1}^{(q)}, \ldots, x_{1}^{(q)}\right.$, $\left.x_{2^{-1}}^{(q)}, x_{2^{\prime-1}-1}^{(q)}, \ldots, x_{s+1}^{(q)}\right)$. If $\operatorname{dif}\left(e_{2}\right) \neq \operatorname{dif}\left(e_{1}\right)$, we assume $e_{2} \in$ $H_{e_{1}}^{0}$ without loss of generality. There is a Hamiltonian cycle, say $\left(y_{1}, y_{2}, \ldots, y_{2^{-1}}, y_{1}\right)$, in $H_{e_{1}}^{0}$ that contains $e_{2}$. Suppose $e_{2}=\left(y_{1}, y_{t+1}\right)$ for some $1 \leq t \leq 2^{r-1}$. A Hamiltonian path in $C B F_{0}(r)$ that contains $e_{1}$ but does not contain $e_{2}$ can be established as $\left(y_{t}, y_{t-1}, \ldots, y_{s}, y_{s}^{(q)}, y_{s+1}^{(q)}, \ldots, y_{2^{x-1}}^{(q)}\right.$, $\left.y_{1}^{(q)}, x_{2}^{(q)}, \ldots, y_{s-1}^{(q)}, y_{s-1}, y_{s-2}, \ldots, y_{1}, y_{2^{r-1}}, y_{2^{r-1}-1}, \ldots, y_{t+1}\right)$ if $s \leq t$, and $\left(y_{t+1}, y_{t+2}, \ldots, y_{s}, y_{s}^{(q)}, y_{s-1}^{(q)}, \ldots, y_{1}^{(q)}, y_{2^{-1}}^{(q)}\right.$, $\left.x_{2^{r-1}-1}^{(q)}, \ldots, y_{s+1}^{(q)}, y_{s+1}, y_{s+2}, \ldots, y_{2^{r-1}}, y_{1}, y_{2}, \ldots, y_{l}\right)$ if $s \geq t+$ 1. When $s=1$, the subpath $\left(y_{2^{-1}}^{(q)}, y_{1}^{(q)}, x_{2}^{(q)}, \ldots, y_{s-1}^{(q)}\right)$ shrinks to a vertex $y_{2^{r-1}}^{(q)}$ and the subpath $\left(y_{s-1}, y_{s-2}, \ldots, y_{1}\right.$, $\left.y_{2^{r-1}}\right)$ shrinks to a vertex $y_{2^{r-1}}$. When $s=2^{r-1}$, the subpath $\left(y_{1}^{(q)}, y_{2^{-1}}^{(q)}, x_{2^{r-1}-1}^{(q)}, \ldots, y_{s+1}^{(q)}\right)$ shrinks to a vertex $y_{1}^{(q)}$ and the subpath $\left(y_{s+1}, y_{s+2}, \ldots, y_{2^{r-1}}, y_{1}\right)$ shrinks to a vertex $y_{1}$. $\square$

The following theorem is the main result of this paper.

Theorem 1. $B F(r)$ with two edge faults contains a Hamiltonian cycle.

Proof. Suppose $\left(u_{1}, v_{1}\right)$ and $\left(u_{2}, v_{2}\right)$ are two edge faults in $B F(r)$, where $\left\{u_{1}, v_{1}\right\} \neq\left\{u_{2}, v_{2}\right\}$. We observe that each edge of $B F(r)$ is either a 0 -edge or a 1 -edge and each 1 -edge ( 0 -edge) connects two distinct 0 -cycles (1-cycles). There are three cases discussed below.

Case 1. $\left(u_{1}, v_{1}\right)$ and $\left(u_{2}, v_{2}\right)$ are 0 -edges. If $\left(u_{1}, v_{1}\right)$ and $\left(u_{2}\right.$, $v_{2}$ ) belong to the same 0 -cycle, say $C_{0}(x)$, then there are two distinct 0 -cycles, say $C_{0}(y)$ and $C_{0}(z)$, that are adjacent to $C_{0}(x)$, and there are two 0 -edges, say $\left(u_{1}^{\prime}, v_{1}\right) \in C_{0}(y)$ and $\left(u_{2}^{\prime}, v_{2}\right) \in C_{0}(z)$, so that $\left(u_{1}, v_{1}\right)$ and $\left(v_{1}, u_{1}\right)$ are a crossing pair between $C_{0}(x)$ and $C_{0}(y)$ and $\left(u_{2}, v_{2}\right)$ and $\left(v_{2}\right.$, $\left.u_{2}\right)$ are a crossing pair between $C_{0}(x)$ and $C_{0}(z)$. Since $(x, y)$ and $(x, z)$ are edges of $C B F_{0}(r)$, Lemma 9 assures a Hamiltonian path in $C B F_{0}(r)$ that contains $(x, y)$ and $(x, z)$. According to Lemma 3, the Hamiltonian path corresponds to a Hamiltonian cycle in $B F(r)$. The Hamiltonian cycle does not contain $\left(u_{1}, v_{1}\right),\left(u_{1}^{\prime}, v_{1}\right),\left(u_{2}, v_{2}\right)$, and $\left(u_{2}^{\prime}, v_{2}\right)$ (refer to the proof of Lemma 3 ).

We then consider that $\left(u_{1}, v_{1}\right)$ and $\left(u_{2}, v_{2}\right)$ belong to two distinct 0-cycles, say $C_{0}(x)$ and $C_{0}(y)$, respectively. If either $\left(u_{1}, v_{2}\right)$ and $\left(v_{1}, u_{2}\right)$ or $\left(u_{1}, u_{2}\right)$ and $\left(v_{1}, v_{2}\right)$ are a crossing pair between $C_{0}(x)$ and $C_{0}(y)$, then a Hamiltonian cycle in $B F(r)$ that does not contain $\left(u_{1}, v_{1}\right)$ and $\left(u_{2}, v_{2}\right)$ can be obtained, similarly, by the aid of Lemma 9 and Lemma 3. If neither $\left(u_{1}, v_{2}\right)$ and $\left(v_{1}, u_{2}\right)$ nor $\left(u_{1}, u_{2}\right)$ and $\left(v_{1}, v_{2}\right)$ are a crossing pair between $C_{0}(x)$ and $C_{0}(y)$, then there are two 0 -cycles, say $C_{0}(x)$ and $C_{0}(y)$, that are adjacent to $C_{0}(x)$ and $C_{0}(y)$, respectively, and there are two 0 -edges, say $\left(u_{1}{ }^{\prime}\right.$, $\left.v_{1}\right) \in C_{0}\left(x^{\prime}\right)$ and $\left(u_{2}^{\prime}, v_{2}\right) \in C_{0}(y)$, so that $\left(u_{1}, v_{1}\right)$ and $\left(v_{1}\right.$, $\left.u_{1}\right)$ are a crossing pair between $C_{0}(x)$ and $C_{0}\left(x^{\prime}\right)$ and $\left(u_{2}, v_{2}\right)$ and $\left(v_{2}, u_{2}\right)$ are a crossing pair between $C_{0}(x)$ and $C_{0}(y)$. A Hamiltonian cycle in $B F(r)$ that does not contain $\left(u_{1}, v_{1}\right)$, $\left(u_{1}^{\prime}, v_{1}\right),\left(u_{2}, v_{2}\right)$, and $\left(u_{2}^{\prime}, v_{2}\right)$ can be obtained, similarly, by the aid of Lemma 9 and Lemma 3.

Case 2. $\left(u_{1}, v_{1}\right)$ and $\left(u_{2}, v_{2}\right)$ are 1-edges. If $\left(u_{1}, v_{1}\right)$ and $\left(u_{2}\right.$, $v_{2}$ ) connect the same pair of 0 -cycles, say $C_{0}(x)$ and $C_{0}(y)$, then they are a crossing pair between $C_{0}(x)$ and $C_{0}(y)$ according to Lemma 2. Since $(x, y)$ is an edge of $C B F_{0}(r)$, Lemma 10 assures a Hamiltonian path in $C B F_{0}(r)$ that does not contain $(x, y)$. Similarly, the Hamiltonian path corresponds to a Hamiltonian cycle in $B F(r)$ that does not contain $\left(u_{1}, v_{1}\right)$ and $\left(u_{2}, v_{2}\right)$. Otherwise, suppose $\left(u_{1}, v_{1}\right)$ connects $C_{0}(x)$ and $C_{0}(y)$ and $\left(u_{2}, v_{2}\right)$ connects $C_{0}(z)$ and $C_{0}(w)$. A Hamiltonian cycle in $B F(r)$ that does not contain $\left(u_{1}, v_{1}\right)$ and $\left(u_{2}, v_{2}\right)$ can be obtained, similarly, by the aid of Lemma 10 and Lemma 3.

Case 3. One of $\left(u_{1}, v_{1}\right)$ and $\left(u_{2}, v_{2}\right)$ is a 0 -edge and the other is a 1 -edge. Without loss of generality, we assume that $\left(u_{1}\right.$, $\left.v_{1}\right)$ is a 0 -edge and $\left(u_{2}, v_{2}\right)$ is a 1-edge. We further assume that $\left(u_{1}, v_{1}\right)$ belongs to $C_{0}(x)$ and $\left(u_{2}, v_{2}\right)$ connects $C_{0}(z)$ and $C_{0}(w)$. There is a 0 -cycle, say $C_{0}(y)$, that is adjacent to $C_{0}(x)$, and there is a 0 -edge, say $\left(u_{1}^{\prime}, v_{1}\right) \in C_{0}(y)$, so that $\left(u_{1}, v_{1}\right)$ and $\left(v_{1}, u_{1}\right)$ are a crossing pair between $C_{0}(x)$ and $C_{0}(y)$. If $\left\{u_{1}, v_{1}\right\} \cap\left\{u_{2}, v_{2}\right\}$ is empty, then $(x, y)$ and $(z, w)$ are two distinct edges of $C B F_{0}(r)$. According to Lemma 11, there is a Hamiltonian path in $C B F_{0}(r)$ that contains $(x, y)$ but does not contain $(z, w)$. Similarly, the Hamiltonian path corresponds to a Hamiltonian cycle in $B F(r)$ that does not contain $\left(u_{1}, v_{1}\right)$ and $\left(u_{2}, v_{2}\right)$.

If $\left\{u_{1}, v_{1}\right\} \cap\left\{u_{2}, v_{2}\right\}$ is not empty, we assume $v_{1}=u_{2} \in$ $C_{0}(w)$ without loss of generality. Hence $C_{0}(x)=C_{0}(w)$. According to Lemma 2 , there is a 1-edge, say $\left(u_{2}^{\prime}, v_{2}\right)$, so that $\left(u_{2}, v_{2}\right)$ and $\left(u_{2}^{\prime}, v_{2}\right)$ are a crossing pair between $C_{0}(x)$ and $C_{0}(z)$, where $u_{2}, u_{2}{ }^{\prime} \in C_{0}(x)$ and $v_{2}, v_{2}{ }^{\prime} \in C_{0}(z)$. If $u_{1} \neq$ 
$u_{2}^{\prime}$, then $(x, y)$ and $(x, z)$ are two distinct edges of $C B F_{0}(2 r)$. A Hamiltonian cycle in $B F(r)$ that does not contain $\left(u_{1}, v_{1}\right)$ and $\left(u_{2}, v_{2}\right)$ can be obtained, similarly, by the aid of Lemma 11 and Lemma 3.

If $u_{1}=u_{2}{ }^{\prime}$, then $C_{0}(y)=C_{0}(z)$ is implied. Recall that $G(r$ - 1) corresponds to a Hamiltonian cycle in $C B F_{1}(r)$. We use $C=\left(x_{1}, x_{2}, \ldots, x_{2^{r-1}}, x_{1}\right)$ to denote the Hamiltonian cycle, where $x_{1}, x_{2}, \ldots, x_{2^{r-1}}$ form a $G(r-1)$. Suppose $C_{1}\left(0 x_{s}\right)$ is the 1-cycle in $B F(r)$ that contains $\left(u_{2}, v_{2}\right)$, where $1 \leq s \leq 2^{r-1}$. According to Lemma $8, C-\left\{x_{s}\right\}$, which is a path of length $2^{r-1}-2$ in $C B F_{1}(r)$, corresponds to a cycle of length $r 2^{r}-2 r$ in $B F(r)$. We use $\hat{C}$ to denote the cycle. The vertex set of $\hat{C}$ and the vertex set of $C_{1}\left(0 x_{s}\right)$ constitute a partition of the vertex set of $B F(r)$. We show in the following that a Hamiltonian cycle in $B F(r)$ that does not contain $\left(u_{1}, v_{1}\right)$ and $\left(u_{2}, v_{2}\right)$ can be obtained by carefully adding the vertices of $C_{1}\left(0 x_{s}\right)$ to $\hat{C}$.

Without loss of generality, we assume $v_{2}=f_{1}\left(u_{2}\right)$ and let $C_{1}\left(0 x_{s}\right)=\left(u_{2}, f_{1}\left(u_{2}\right)\left(=v_{2}\right), f_{1}^{2}\left(u_{2}\right), \ldots, f_{1}^{2 r-1}\left(u_{2}\right), f_{1}^{2 r}\left(u_{2}\right)\right.$ $\left.\left(=u_{2}\right)\right)$. For $1 \leq t \leq r$, let $w_{t}=f_{0}\left(f_{1}^{2 t-1}\left(u_{2}\right)\right) \in \hat{C}$ and $w_{i}^{\prime}=$ $f_{0}^{-1}\left(f_{1}^{2 t}\left(u_{2}\right)\right) \in \hat{C}$. It is not difficult to see that $\left(f_{1}^{2 t-1}\left(u_{2}\right), w_{t}\right)$ and $\left(f_{1}^{2 t}\left(u_{2}\right), w_{t}\right)$ are a crossing pair between $C_{1}\left(0 x_{s}\right)$ and $\hat{C}$. Since $\left\{w_{2 p-1}, w_{2 p}\right\} \cap\left\{w_{2 q-1}, w_{2 q}\right\}$ is empty for all $p \neq q$, a Hamiltonian cycle in $B F(r)$ can result if $\left(f_{1}^{2 t-1}\left(u_{2}\right), w_{1}\right),\left(f_{1}^{2 t}\left(u_{2}\right), w_{r}\right)$, and $\left(f_{1}^{2 t-1}\left(u_{2}\right)\right.$, $\left.f_{1}^{2 t}\left(u_{2}\right)\right)$ are added to $\hat{C}$ and $\left(w_{t}, w_{t}\right)$ is removed from $\hat{C}$ for all $1 \leq t \leq r$. Since $\left(u_{2}, v_{2}\right)=\left(f_{1}^{2 r}\left(u_{2}\right), f_{1}\left(u_{2}\right)\right) \notin$ $\left\{\left(f_{1}^{2 t-1}\left(u_{2}\right), f_{1}^{2 t}\left(u_{2}\right)\right) \mid 1 \leq t \leq r\right\}$, the Hamiltonian cycle does not contain $\left(u_{2}, v_{2}\right)$. Moreover, the Hamiltonian cycle does not contain $\left(u_{1}, v_{1}\right)$ because $\left(v_{1}, u_{1}\right) \notin\left\{\left(f_{1}^{2 t-1}\left(u_{2}\right), w_{1}\right)\right.$, $\left.\left(f_{1}^{2 t}\left(u_{2}\right), w_{1}\right) \mid 1 \leq t \leq r\right\}$, as explained below.

We have $f_{0}\left(v_{1}\right)=u_{1}$ because $v_{2}=f_{1}\left(u_{2}\right)$ and $\left(u_{2}, v_{2}\right)(=$ $\left.\left(v_{1}, u_{1}^{\prime}\right)\right)$ and $\left(u_{2}^{\prime}, v_{2}\right)\left(=\left(u_{1}, v_{1}^{\prime}\right)\right)$ are a crossing pair between $C_{0}(x)$ and $C_{0}(z)\left(=C_{0}(v)\right)$. Since $f_{1}^{c}\left(u_{2}\right)=f_{1}^{c}\left(v_{1}\right)$ $\neq f_{0}\left(v_{1}\right)=u_{1}$ for all $1 \leq c \leq 2 r$ and $f_{1}^{c}\left(u_{2}\right)=v_{1}$ only when $c$ $=2 r$, we have $\left(v_{1}, u_{1}\right) \notin\left\{\left(f_{1}^{2 t-1}\left(u_{2}\right), w_{t}\right),\left(f_{1}^{2 t}\left(u_{2}\right), w_{l}\right) \mid\right\}$ $\leq t \leq r\}$ if $u_{1} \neq w_{r}{ }^{\prime}$. The latter is true because $w_{r}{ }^{\prime}=$ $f_{0}^{-1}\left(f_{1}^{2 r}\left(u_{2}\right)\right)=f_{0}^{-1}\left(u_{2}\right)=f_{0}^{-1}\left(v_{1}\right) \neq f_{0}\left(v_{1}\right)=u_{1}$.

\section{Conclusion and discussion}

In this paper, we showed that $B F(r)$ with two edge faults contains a Hamiltonian cycle. Since $C B F_{0}(r)$ is isomorphic to the $r$-dimensional hypercube, Lemma 9 , Lemma 10, and Lemma 11 are also valid for the latter. We suspect that Theorem 1 can be generalized as follows: An $r$-dimensional $k$-ary butterfly graph with $2 k-2$ edge faults contains a Hamiltonian cycle. However, the proof seems very hard.

\section{References}

[1] S. B. Akers and B. Krishnamurthy, "A grouptheoretic model for symmetric interconnection networks," IEEE Transactions on Computers, vol. 39, no. 4, pp. 555-566, 1989.

[2] F. Annexstein, M. Baumslag, and A. L. Rosenberg, "Group action graphs and parallel architectures," SIAM Journal on Computing, vol. 19, no. 3, pp. 544-569, 1990.

[3] D. Barth and A. Raspaud, "Two edge-disjoint Hamiltonian cycles in the butterfly graph," Information Processing Letters, vol. 51, pp. 175-179, 1994.

[4] J.-C. Bermond, E. Darrot, O. Delmas, and S. Perennes, "Hamilton circuits in the directed wrapped butterfly network," Discrete Applied Mathematics, vol. 84, pp. 2 i-42, 1998.

[5] J.-C. Bermond, E. Darrot, O. Delmas, and S. Perennes, "Hamilton cycle decomposition of the butterfly network," Parallel Processing Letters, vol. 8, no. 3, pp. 371-385, 1998.

[6] P. Fragopoulou and S. G. Akl, "A parallel algorithm for computing Fourier transform on the star graph," Proceedings of the International Conference on Parallel Processing, 1991, pp. 100-106.

[7] J. Y. Jou and J. A. Abraham, "Fault-tolerant FFT networks," IEEE Transactions on Compuers, vol. 37 , no. 5, pp. 548-561, 1983.

[8] S. Lakshmivarahan and S. K. Dhall, Analysis and Design of Parallel Algorithms: Arithmetic and Matrix Problems, McGram-Hill, New York, NY, 1990.

[9] S. Lakshmivarahan, J. S. Jwo, and S. K. Dhall, "Embedding of cycles and grids in star graphs," Journal of Circuits, Systems, and Computers, vol. 1, no. 1, pp. 43-74, 1991.

[10] F. T. Leighton, Introduction to Parallel Algorithms and Architecture: Arrays-Trees.Hypercubes, Morgan Kaufman, San Mateo, CA, 1992.

[11] Y. Saad and M. H. Schultz, "Topological properties of hypercubes," IEEE Transactions on Computers, vol. 37, no. 7, pp. 867-872, 1988.

[12] S. A. Wong, "Hamilton cycles and paths in butterfly graphs," Networks, vol. 26, pp. 145-150, 1995. 